\title{
Análisis de la ultrafiltración media por sesión de los pacientes en una unidad de hemodiálisis
}

\author{
Mónica Pereira-García ${ }^{1}$, Paula Manso-del-Real' ${ }^{1}$, Raúl Fernández-Prado² ${ }^{2}$ Alejandro Avello-Escribano² ${ }^{2}$ Emilio \\ González-Parra ${ }^{2}$
}

${ }^{1}$ Fundación Renal Iñigo Álvarez de Toledo-Hospital Universitario Fundación Jiménez Díaz. Madrid. España

${ }^{2}$ Hospital Universitario Fundación Jiménez Díaz. Madrid. España

Como citar este artículo:

Pereira-García M, Manso-del-Real P, Fernández-Prado R, Avello-Escribano A, González-Parra E. Análisis de la ultrafiltración media por sesión de los pacientes en una unidad de hemodiálisis.

Enferm Nefrol. 2020 Abr-Jun;23(2):192-7

\section{Resumen}

Introducción: En los pacientes en hemodiálisis (HD), el ajuste erróneo del peso ideal puede llevarlos a la sobrecarga de líquido que puede desembocar en episodios de insuficiencia cardiaca o a la inversa, a una situación de bajo peso generando hipotensiones que pueden originar cardiopatía isquémica. Se estima como adecuada una ultrafiltración (UF) máxima de $10 \mathrm{ml} / \mathrm{kg} / \mathrm{h}$, valor por encima del cual está demostrado el aumento de la mortalidad.

Objetivos: Analizar que tasa de ultrafiltración media usamos en nuestra unidad, y conocer qué porcentaje de pacientes sobrepasaban la ultrafiltración máxima recomendada.

Material y Método: Realizamos un estudio observacional, prospectivo, en 58 pacientes en hemodiálisis, analizando la tasa de ultrafiltración expresada en $\mathrm{ml} /$ $\mathrm{kg} / \mathrm{h}$ por sesión durante 6 meses. Se definió una tasa de UF $>10 \mathrm{ml} / \mathrm{kg} / \mathrm{h}$ como punto de corte para, según los criterios actuales de tasa adecuada, determinar que pa-

\section{Correspondencia:}

Mónica Pereira García.

Email: mpereira@friat.es cientes presentaban una UF superior a $10 \mathrm{ml} / \mathrm{Kg} / \mathrm{h}$ en más de un $25 \%$ de las sesiones.

Resultados: Durante el periodo de estudio la tasa media de UF de todos los pacientes fue de 8,78 $2,76 \mathrm{ml} / \mathrm{kg} / \mathrm{h}$, aunque el porcentaje de sesiones por paciente con tasa de UF superior a $10 \mathrm{ml} / \mathrm{kg} / \mathrm{h}$ fue de un $35,9 \pm 29,74 \%$.

Conclusión: Un porcentaje alto de pacientes presentan tasas de UF por encima de los valores recomendados. Se han de buscar estrategias para minimizar esta situación, donde la educación sanitaria sobre alimentación y el ajuste individualizado de las sesiones de diálisis son aspectos fundamentales.

PALABRAS CLAVE: tasa de ultrafiltración; peso seco; hemodiálisis; morbimortalidad; atención de enfermería.

\section{Analysis of the average ultrafiltration rate per session of patients in a hemodialysis unit}

\section{Abstract}

Introduction: In haemodialysis (HD) patients, the wrong adjustment of the ideal weight can lead to fluid overload, which can cause episodes of heart failure or, conversely, to a low weight situation, generating 
hypotension that triggers ischemic heart disease. A maximum ultrafiltration (UF) of $10 \mathrm{ml} / \mathrm{kg} / \mathrm{hr}$ is considered adequate. A higher value is associated with an increase in mortality.

Objectives: To analyse the average ultrafiltration rate used in the study centre, and to know what percentage of patients exceeded the maximum recommended ultrafiltration.

Material and Method: An observational, prospective study in 58 haemodialysis patients was carried out, analysing the ultrafiltration rate expressed in $\mathrm{ml} / \mathrm{kg} / \mathrm{hr}$ per session for 6 months. A UF rate $>10 \mathrm{ml} / \mathrm{kg} / \mathrm{hr}$ was defined as the cut-off point, according to the current criteria of adequate rate, to determine which patients had a UF greater than $10 \mathrm{ml} / \mathrm{kg} / \mathrm{hr}$ in more than $25 \%$ of the sessions.

Results: During the study period, the average UF rate of all patients was $8.78 \pm 2.76 \mathrm{ml} / \mathrm{kg} / \mathrm{hr}$, although the percentage of sessions per patient with a UF rate greater than $10 \mathrm{ml} / \mathrm{kg} / \mathrm{hr}$ was $35.9 \pm 29.74 \%$.

Conclusion: A high percentage of patients present UF rates above the recommended values. Strategies to decrease values must be sought, with health education on diet and individualized adjustment of dialysis sessions being fundamental aspects.

KEYWORDS: ultrafiltration rate; dry weight; haemodialysis; morbidity and mortality; nursing care.

\section{Introducción}

Los pacientes con insuficiencia renal presentan una mayor morbimortalidad cardiovascular, en relación con la población general ${ }^{1,2}$.

Diferentes estudios, con una amplia muestra de pacientes, han demostrado que la insuficiencia renal es un factor de mortalidad, morbilidad e ingresos hospitalarios por causa cardiovascular. Go y cols, analizaron el riesgo cardiovascular de más de un millón de personas $y$, observaron que es superior en aquellos con insuficiencia renal y que este riesgo se incrementa a medida que desciende el filtrado glomerular ${ }^{1}$. Múltiples estudios poblacionales objetivan esta evidencia, entre los que destacan algunos como los de Hoorn².

Se ha comprobado que entre el $30-40 \%$ de los pacientes con insuficiencia renal crónica, presentan cardiopatía isquémica en ausencia de estenosis hemodinamicamente significativas de las arterias coronarias subepicárdicas ${ }^{3}$. Los mecanismos potencialmente implicados en esta situación son dos: que las placas ateroescleróticas de los enfermos con insuficiencia renal crónica se calcifican con mayor frecuencia que la población general, y que la capa media de la pared arterial está engrosada. Estas alteraciones aumentan la rigidez de la aorta y las grandes arterias, facilitando la elevación de la presión sistólica y la presión del pulso, lo que lleva a una hipertrofia ventricular izquierda y un deficiente llenado coronario durante la diástole ${ }^{4}$.

El riesgo cardiovascular es especialmente alto en los pacientes en diálisis. A los factores asociados a la enfermedad renal se añaden los específicos de la técnica de diálisis. En los pacientes en diálisis peritoneal el factor diferenciador es la sobrecarga de volumen constante. En hemodiálisis (HD), el ajuste erróneo del peso ideal es el principal motivo ya que puede llevar a nuestros pacientes a dos situaciones contrapuestas, por un lado, a la sobrecarga de líquido que puede desembocar en episodios de insuficiencia cardiaca con un gran riesgo vital, o a la inversa, a una situación de bajo peso generando hipotensiones que pueden originar cardiopatía isquémica igual de peligrosa 5 . Por este motivo la técnica de HD exige una individualización constante. Ningún paciente se parece a otro. Debemos calcular el peso ideal de una manera fiable, periódica, concreta y protocolizada. Siendo enfermería parte fundamental en este aspecto por su interacción diaria con el paciente, ya que habitualmente somos conocedoras de la variación diaria de peso, la ingesta habitual y la situación personal del paciente.

Pero dentro de la individualización de la técnica de $H D$, es la ultrafiltración (UF) del paciente lo que exige prudencia. Si hacemos una UF horaria excesiva la mayoría de los pacientes tendrán efectos secundarios tales como hipotensión intradiálisis o calambres, y algunos pacientes pérdida de conocimiento. Esas hipotensiones ocasionan un daño cardiaco que puede ser fatal para el paciente ${ }^{6}$.

Esta UF máxima debe de ser individualizada también, aunque en la mayoría de las unidades esta UF está establecida entre $700-1000 \mathrm{ml} / \mathrm{h}$ como dato estándar sin individualización de la practica ${ }^{7}$. Recientemente se ha visto que una UF mayor de $13 \mathrm{ml} / \mathrm{Kg} / \mathrm{h}$ se relaciona con mayor mortalidad ${ }^{8}$. Otros grupos lo han establecido en $10 \mathrm{ml} / \mathrm{Kg} / \mathrm{h}^{9}$. Estando ambos datos de UF máxima directamente relacionados con el peso específico de cada 
paciente. Esto obliga a tomar medidas relacionadas con el ajuste de $\mathrm{ml} / \mathrm{h}$ adecuados para cada individuo ya sea variando el tiempo, numero de sesiones semanales, control de la ingesta, adherencia terapéutica a las recomendaciones y un largo etcétera para no sobrepasar la UF horaria máxima recomendada.

Como objetivo de estudio hemos analizado la tasa de UF horaria corregida por el peso con el fin de determinar cómo se ajusta nuestra práctica habitual a las recomendaciones actuales, y definir la implicación de la enfermería sobre las posibilidades de mejora para impedir tasas elevadas de UF.

\section{Material y Método}

Estudio observacional, prospectivo, realizado entre el 1 enero 2018 y el 30 junio 2018, en la unidad de HD del Hospital Universitario Fundación Jiménez Díaz en Madrid. Se incluyeron todos los pacientes adultos que llevaban un mínimo de tres meses en programa de HD en nuestro centro, independientemente del número de sesiones semanales, y que voluntariamente dieron su consentimiento para participar en el estudio. Se excluyeron los pacientes que recibieron tratamiento de HD de forma aguda y aquellos pacientes que no permanecieron en tratamiento con HD durante todo el periodo de seguimiento.

Como variables de estudio se han recogido datos demográficos como la edad, sexo, y factores de riesgo cardiovascular (vasculopatía periférica, insuficiencia cardíaca y cardiopatía isquémica). Se registraron otras características de diálisis como la duración de las sesiones, peso seco, volumen UF total, UFR en $\mathrm{ml} / \mathrm{kg} / \mathrm{h}$, porcentaje reducción de peso, tiempo en diálisis, acceso vascular, tensión arterial sistólica pre(pre-TAS) y post(post-TAS) HD.

Los datos relativos a tasa de UF horaria durante el periodo de estudio se recogieron del registro del programa informático Nefrosoft ${ }^{\circledast}$ de nuestra unidad, de manera que recogimos el peso pre-HD y post-HD, así como el peso ideal pautado o peso seco. Para el cálculo del peso seco, cada 2 meses de realizaba una bioimpedancia mediante el monitor BCM (Body Composition Monitor) de Fresenius. Todos estos datos se recogieron en todas las sesiones del periodo de estudio.

Se calculó la UF total, UF horaria y UF horaria corregida para el peso seco durante cada sesión. Calculamos la UF horaria en mililitros, dividiendo la UF total por las horas de diálisis, y para ajustarlo al peso se dividió por el peso seco. Los datos se volcaron de una manera directa en una tabla Excel.

Como valor de referencia se estableció una tasa de UF $>10 \mathrm{ml} / \mathrm{kg} / \mathrm{h}$, calculando en cuantas sesiones de HD se superaban estos valores. Para evitar incluir a los pacientes que de forma esporádica cumplieran esta condición, se decidió establecer como punto de corte a aquellos pacientes que en al menos un $25 \%$ de las ocasiones hubieran tenido una tasa de UF>10 ml/Kg/h.

Se calculó el porcentaje de reducción del peso en cada sesión, para ello se dividió el peso perdido por el paciente en cada sesión por el peso seco del paciente, multiplicado el resultado por 100.

Los episodios de calambres, hipotensión y vómitos se usan como un indicador de tolerancia a la sesión de diálisis y suelen indicar UF excesiva. En este estudio no hemos analizado estos eventos.

Los pacientes firmaron un consentimiento informado donde se explicaba la información relativa al estudio. Toda la investigación clínica se realizó de acuerdo con los principios éticos para investigación médica en seres humanos y la Directiva de ensayos clínicos de la Unión Europea (2001/20/CE) expresados en la Declaración de Helsinki.

Para el análisis estadístico, las variables categóricas fueron expresadas como frecuencias y porcentajes, y las variables continuas como medias y derivaciones estándar [SD] cuando éstas seguían una distribución gaussiana/normal, o como medianas y rangos intercuartílicos [IQR= percentil 25th-75th] cuando la distribución se alejaba de la normalidad. Los porcentajes fueron comparados utilizando el test de Chi-cuadrado [X2] o el test exacto de Fisher; las medias por el t-test y las medianas mediante el test de Wilcoxon para datos independientes. La diferencia significativa fue establecida con una $p<0,05$.

\section{Resultados}

Se incluyeron en el estudio un total de 56 pacientes con una edad media $72,5 \pm 14,52$ años, de los cuales eran mujeres un $27,1 \%$. El peso ideal o peso seco basal medio fue de $69,99 \pm 13,23 \mathrm{~kg}$ y el volumen de UF total medio durante toda la sesión de diálisis fue de $2266,78 \pm 678,84 \mathrm{ml}$. 
La tasa media de UF $(\mathrm{ml} / \mathrm{kg} / \mathrm{h})$ de todos los pacientes durante el periodo de estudio fue de $8,78 \pm 2,76 \mathrm{ml} /$ $\mathrm{kg} / \mathrm{h}$. Cuando analizamos el porcentaje de sesiones en cada paciente en las que llevo a cabo una tasa de UF $\geq 10 \mathrm{ml} / \mathrm{kg} / \mathrm{h}$, observamos este hecho ocurría en un $35,9 \pm 29,74 \%$ de las mismas. Sin embargo, cuando analizamos el porcentaje de pacientes con tasa de UF $\geq 10 \mathrm{ml} / \mathrm{kg} / \mathrm{h}$ en al menos un $25 \%$ de las sesiones vemos que esta situación se producía en el 50,8\%.

El porcentaje de reducción medio del peso en cada sesión de diálisis fue del $2,73 \pm 0,83 \%$.

En la Tabla 1 se puede ver la comparación de los valores de presión arterial sistólica pre y post hemodiálisis, así como la diferencia entre estos valores, en los pacientes que tuvieron tasas de UF $\geq 10 \mathrm{ml} / \mathrm{kg} / \mathrm{h}$ en al menos un $25 \%$ de las sesiones de HD y en lo que ocurrió en menos de un $25 \%$ de las sesiones, no encontrándose diferencias significativas entre ambos grupos.

Tabla 1. Comparación de los valores de presión arterial sistólica pre y post hemodiálisis.

\begin{tabular}{|c|c|c|c|}
\hline Variable & Grupo 1 & Grupo 2 & $\mathbf{p}$ \\
\hline TAS pre ${ }^{1}$ & $14 \pm 20,0$ & $133,9 \pm 21,5$ & 0,186 \\
\hline TAS post ${ }^{1}$ & $132 \pm 18,2$ & $125,5 \pm 19,2$ & 0,173 \\
\hline $\begin{array}{l}\text { \% de reducción } \\
\text { de } \mathrm{TAS}^{1}\end{array}$ & $-5,89 \pm 8,63$ & $-5,85 \pm 9,44$ & 0,984 \\
\hline
\end{tabular}

Grupo 1: pacientes con tasas de $U F \geq 10 \mathrm{ml} / \mathrm{kg} / \mathrm{h}$ en $\leq 25 \%$ de las sesiones de HD. Grupo 2: pacientes con tasas de $U F \geq 10 \mathrm{ml} / \mathrm{kg} / \mathrm{h}$ en $>25 \%$ de las sesiones de HD. TAS: tensión arterial sistólica; TAD: tensión arterial diastólica.

${ }^{1}$ Media \pm desviación estándar.

\section{Discusión}

La mala tolerancia durante la sesión de HD se debe fundamentalmente a desequilibrios hidroelectrolíticos, manifestándose con cambios en la tensión arterial (TA $)^{10}$.

La TA se suele usar como marcador de sobrecarga de líquidos, de manera que TA por encima de $160 \mathrm{mmHg}$ suelen expresar sobrecarga ${ }^{11}$. Existe la idea de que la hipertensión en un paciente en HD suele ser debida a sobrecarga de agua. La bajada de la TA depende, entre otros factores, de la extracción de líquido y la capacidad del paciente para aumentar las resistencias periféricas. Es conocido que el paciente en HD suele tener alteraciones en el sistema simpático que impide la vasoconstricción.
La pérdida rápida de agua corporal, cuando no hay capacidad de vasoconstricción provoca la bajada de la TA; de ahí la importancia que en los últimos años está suponiendo la utilización de herramientas como bioimpedancia, que nos ayuda a estimar esta sobrecarga de agua ${ }^{12}$.

Las hipotensiones durante la sesión de hemodiálisis aumentan el riesgo de daño cardiaco, neurológico e inciden directamente sobre la mortalidad total, por lo que deben de ser evitadas ${ }^{13}$, además aceleran la pérdida de función renal residual ${ }^{14}$. Por otro lado, se ha de ser cauto, porque el realizar una UF menor a la necesitada, podría ocasionar una sobrecarga de líquido y favorecer el desarrollo de un edema agudo de pulmón.

En nuestro estudio hemos analizado los valores de TA pre y post hemodiálisis, así como los cambios producidos entre los mismos, no encontrando diferencias entre los dos grupos estudiados; aunque debemos tener en cuenta que no se estudiaron las hipotensiones intradiálisis. Una posible explicación sería que la población estudiada tiene una edad media no muy alta, lo que podría hacernos pensar que la respuesta a la ultrafiltración no se manifestara clínicamente. Habría sido interesante conocer si hubo hipotensiones intradiálisis y ver su posible repercusión.

La UF total es un parámetro muy utilizado, pero no tiene en cuenta el tamaño del paciente ni su composición corporal, sin embargo, el utilizar la tasa de UF calculada por peso ayuda comprender mejor estos riesgos. Diversos trabajos sugieren como un valor de referencia una UF calculada por el peso entre 10 y $13 \mathrm{ml} / \mathrm{kg} / \mathrm{h}^{10,11}$. Este concepto es interesante al tener en cuenta por primera vez la importancia de la cantidad relativa de UF dependiendo del peso del paciente.

En el presente estudio hemos objetivado que la tasa media de UF calculada por el peso de todos los pacientes fue de $8,78 \pm 2,76 \mathrm{ml} / \mathrm{kg} / \mathrm{h}$. Si solo analizamos esos datos nuestra unidad reúne una adecuada tasa de UF, de acuerdo con los resultados considerados como seguros ${ }^{11}$. Sin embargo, un 35\% de nuestros pacientes tienen tasa media de UF $>10 \mathrm{ml} / \mathrm{Kg} / \mathrm{h}$ y un $50,8 \%$ superan dicha tasa en el $25 \%$ de las sesiones de diálisis estudiadas. Nos parece relevante este dato, pues es un alto número de diálisis con UF elevadas.

Esta variable exige una individualización de la sesión dependiendo de la UF para evitar aumento morbimortalidad $^{15}$. Cuando es preciso, el paciente debe incrementar el tiempo de diálisis para ajustar la UF, especialmente durante el periodo largo inter diálisis. 
El papel de la enfermería de HD es muy destacado en el control de la UF. La tasa de UF por peso debe de ser conocida por enfermería pues indica un incremento del riesgo probable de mortalidad, a la vez que este conocimiento puede ayudar a orientar al nefrólogo sobre un cambio de pauta de diálisis.

En los casos en los que la tasa de UF sea elevada, es recomendable acordar con los facultativos modificar el tiempo de diálisis diaria y/o semanal. Es importante, desde la intervención del personal de enfermería, trabajar conjuntamente con el paciente en el control de la ingesta hídrica y adecuar la ganancia de peso interdiálisis, mediante la formación y autocuidado de nuestros pacientes a través de la educación para la salud. Los pacientes cuya tasa de UF es alta y precisan de incrementos repetidos del tiempo de diálisis requieren una mayor vigilancia.

Es imprescindible la necesidad de individualizar la pauta de los pacientes en cada sesión y ajustar el tiempo de diálisis calculando la tasa de UF horaria para evitar eventos adversos y reducir la mortalidad.

Como limitaciones principales de nuestro estudio encontramos, el bajo número de pacientes y no haber observado la repercusión clínica de la elevada UF, incluida la mortalidad.

A partir de los resultados encontrados podemos apreciar que el número de pacientes con ultrafiltraciones horarias elevadas es más alto de lo esperado. El conocimiento de este aspecto es importante para modificar la pauta de diálisis, los hábitos de vida y adherencia terapéutica, con el fin de mejorar o disminuir la morbi-mortalidad de los pacientes en tratamiento en hemodiálisis.

Recibido: 05-02-20

Revisado: 15-02-20

Modificado: 05-04-20

Aceptado: 25-04-20

\section{Bibliografía}

1. Go AS, Hertow GM, Fan D, McCulloch CE, Hsu CY. Chronic kidney disease and the risks of death, cardiovascular events, and hospitalization. N.Eng. J. Med. 2004;351:1296-305.

2. A Abramson JL, Jurkovitz CT, Vaccarino $V$, Weintraub WS, McClellan W. Chronic kidney disease, anemia, and incident stroke in a middle-aged, community-based population: the ARIC Study.. Kidney Int. 2003;64:610-5.

3. Tyralla K, Amann K: Morphology of the heart and arteries in renal failure. Kidney Int. 2003; 63: (Suppl 84):S80-3.

4. Safar ME, London GM, Plante GE: Arterial stiffness and kidney function. Hipertensión 2004;43: 163-8.

5. Burton J0, Jefferies HJ, Selby NM, McIntyre CW. Hemodialysis-induced cardiac injury: determinants and associated outcomes. Clin J Am Soc Nephrol. 2009;4(5):914-20.

6. Preciado $P$, Zhang $H$, Thijssen $S$, Kooman JP, van der Sande FM, Kotanko P. All-cause mortality in relation to changes in relative blood volume during hemodialysis. Nephrol Dial Transplant. 2019;34(8):1401-8.

7. Kuipers J, Verboom LM, Ipema KJR, Paans W, Krijnen WP, Gaillard CAJM, et al, The Prevalence of Intradialytic Hypotension in Patients on Conventional Hemodialysis: A Systematic Review with Meta-Analysis. AmJ Nephrol. 2019; 49(6):497-506.

8. Flythe JE, Xue $H$, Lynch KE, Curhan GC, BruneIli SM. Association of mortality risk with various definitions of intradialytic hypotension. J Am Soc Nephrol. 2015;26(3):724-34.

9. Assimon MM, Wenger JB, Wang L, Flythe JE. UItrafiltration Rate and Mortality in Maintenance Hemodialysis Patients. Am J Kidney Dis. 2016; 68(6):911-22.

10. Agarwal R, Weir MR. Dry-weight: a concept revisited in an effort to avoid medication-directed approa- 
ches for blood pressure control in hemodialysis patients. Clin J Am Soc Nephrol. 2010;5:1255-260.

11. Park J, Rhee CM, Sim JJ, Kim YL, Ricks J, Streja $E$, et al. A comparative effectiveness research study of the change in blood pressure during hemodialysis treatment and survival. Kidney Int. 2013;84:795802.

12. Davies SJ, Davenport A. The role of bioimpedance and biomarkers in helping to aid clinical decision-making of volume assessments in dialysis patients. Kidney Int. 2014;86:489-96.

13. Daugirdas JT. Intradialytic hypotension and splanchnic shifting: Integrating an overlooked mechanism with the detection of ischemia-related signals during hemodialysis. Semin Dial. 2019;32(3):243-7.

14. Lee Y, Okuda Y, Sy J, Kim SR, Obi Y, Kovesdy CP, et al. Ultrafiltration Rate Effects Declines in Residual Kidney Function in Hemodialysis Patients. Am J Nephrol. 2019:1-8.

15. Chou JA, Kalantar-Zadeh K. Volume Balance and Intradialytic UItrafiltration Rate in the Hemodialysis Patient. Curr Heart Fail Rep. 2017;14(5):421-7.

Este artículo se distribuye bajo una Licencia Creative Commons Atribución-NoComercial 4.0 Internacional. https://creativecommons.org/licenses/by-nc/4.0/

\section{Open Access c) (1) \&}

